\title{
Near Infrared Spectroscopy (NIRS) in the clinical setting - An adjunct to monitoring during diagnosis and treatment
}

\author{
R.E. Gagnon and A.J. Macnab* \\ Department of Pediatrics, University of British Columbia, Vancouver, Canada
}

\begin{abstract}
Since clinical near infrared spectrophotometers (NIRS) first became commercially available in the early 1990's there have been more than two thousand related peer reviewed reports in the medical literature. These encompass a wide range of human and animal trials that have been directed at validating the principles, methods, and algorithms underlying the technology, as well as demonstrating its potential for specific clinical uses such as detecting concealed bleeding, onset of hypoxia, progress of ischemia, and tissue oxygenation status within human brain, muscles, organs, and tumours. In addition to its standard use as a monitor of patterns of change in the concentrations of oxygenated, and de-oxygenated hemoglobin residing in blood, NIRS has also been used to monitor patterns of change in the redox status of the cellular respiration enzyme, cytochrome coxidase $\left(C y t a_{2} a_{3}\right)$ which utilizes the oxygen diffused from the blood. Accompanied by a tracer bolus of near infrared absorbing dye, NIRS has also been used to measure the proportional blood flow and blood volume transiting organs. NIRS has been used in conjunction with PET, fMRI, BOLD-fMRI, TCD, vascular flowmetry, MRS, NMR, plethysmography, $\mathrm{PO}_{2}$ histography, EEG, ECG, EMG, SSEP, MEP, MEG, and standard bedside monitoring devices. Herein we summarize the history, technique, algorithms, methods and advances of clinical NIRS.
\end{abstract}

Keywords: Spectrophotometry, cytochrome, hemoglobin, adenosine triphosphate, phosphorylation, mitochondria

\section{Evolution}

Many people have a childhood memory of placing their palm over a lit flashlight to be amazed by the transformed reddish glow of their hand. Its not surprising then, that one of the earliest reports of tissue spectroscopy was that by Von Vierordt in 1876 regarding the spectroscopy of blood in the human hand. Modern clinical near infrared spectrophotometry (NIRS) combines the established 18th and 19th century Beer-Lambert principles of light attenuation, arising from chromophore concentration and path length, with the current principles of photon diffusion, spatial resolution, and time-of-flight theories [1]. It is plausible that the first clinical application of NIRS took place circa 1940 at the Sloan-Kettering Institute for Cancer Research to study the metabolism of steroids [2]. In 1996, The Royal Society (London) held the first public seminar devoted entirely to in vivo clinical NIRS [3]. Today, some 130 years since its inception, there are more than 2200 relevant peer reviewed articles archived in the medical literature.

The emergence of modern clinical NIRS is attributed to Norris in 1977 who described the application of NIRS to the study of in situ human tissues, and to Jobsis, also in 1977, who described the application of NIRS to the study of cerebral metabolism as related to cytochrome c oxidase, also known

\footnotetext{
${ }^{*}$ Corresponding author: Dr. Andrew Macnab, The Bladder Care Centre, Suite 1B, Koerner Pavilion, 2211 Wesbrook Mall, UBC Campus Hospital, Vancouver, BC, Canada, V6T 2B5. Tel.: +1 604822 7616; Fax: +1 604822 7591; E-mail: roy.gagnon @ telus.net.
} 
as cytochrome $\mathrm{a}, \mathrm{a}_{3}(\mathrm{Cyt})$. Coincident with the many developments in NIRS technology there were other critical breakthroughs in supporting sciences, including: publication of the absorption spectrum of hemoglobin (c1943), the measurement of cerebral blood flow (c1948), publication of the absorption spectrum of mammalian cytochrome c oxidase (c1961), invention of the optical laser (694 nm) (c1960), a description of electron transport level phosphorylation coupled with the proton motive force that enables adenosine triphosphate (ATP) synthesis (c1961), description of electron transfers within cytochrome c oxidase (c1992), description of proton pumping within cytochrome c oxidase (c1994) and derivation of the ATP synthase protein's activity/response cycle (c2000).

Although the use of near infrared devices for clinical pulse oximetry based solely on hemoglobin measurements was established in 1980, the use of NIRS for transcutaneous penetration incorporating Cyt measurement in conjunction with hemoglobin measurements began clinically in the later 1980's with the need to resolve nuclear magnetic resonance (NMR) (MRS) anomalies in neonatal cerebral data collections. Magnetic resonance was able to measure cerebral adenosine triphosphate (ATP) activity, but confirmation of Cyt activity, as provided by NIRS, was needed to aid clinical interpretation. However, it was not until the mid 1990's that a $2.8 \AA$ mapping of the metal sites of oxidized Cyt could be made, in order to elucidate the pathways of electron transfer and proton pumping within the oxidase, thereby affirming the co-operative relationship between ATP and Cyt [4].

Today, there are numerous commercial versions of clinical NIRS devices available for non-invasive transcutaneous sampling. Some use only one wavelength of emitted light, many use two wavelengths, a few use three wavelengths, one or two use four or five wavelengths, and several allow selection of 2 to 16 wavelengths (Fig. 1). Some units are handheld while others are full console models with 2 to 32 data channels for multiple site collections. Those using four or more wavelengths often, but not always, include Cyt measurement in their features.

\section{Applications}

The non-invasive and bedside utility of NIRS first made itself attractive for brain monitoring of sick new born infants, a group that it was believed would most greatly benefit from introduction of this new technology [5]. Since then it has been used in cohorts of all ages (Fig. 2). The early brain studies were soon followed by muscle oxygenation trials. Over the ensuing decade, NIRS demonstrated its potential for monitoring of liver, breast, spine, and tumors in humans; and, demonstrated that in animal models it could also monitor the lung, testis, spinal cord, kidney, heart, bowel, and splanchnic region.

In the clinical setting, the non-invasive and portable bedside attributes of NIRS has made it particularly useful for studying the very prematurely born afflicted with brain lesions, apnea, and undeveloped autoregulation. It has been used to monitor childhood mitochondrial disease states, encephalopathy, epilepsy, and type 1 diabetes, as well as to monitor those infants having responses to noxious stimuli and suctioning as a consequence of being mechanically ventilated. It has been useful in examining the olfactory, auditory, visual, and language senses in newborns and adults. In adults, NIRS has been used to gain new insights into recovery from depression, schizophrenia, impaired cognitive function, head injury, stroke, cancer, sepsis and obstructive sleep apnea. Apart from brain studies, NIRS has frequently been used in adults to study muscle physiology, muscle oxygen extraction, and muscle related sports injuries.

In the surgical setting, NIRS is used to monitor cerebral oxygenation status during prolonged anesthesia where organs other than the brain are being operated on, as well as being used during brain surgery. These include routine cardiac surgery procedures where circulatory intervention, hypothermia, 


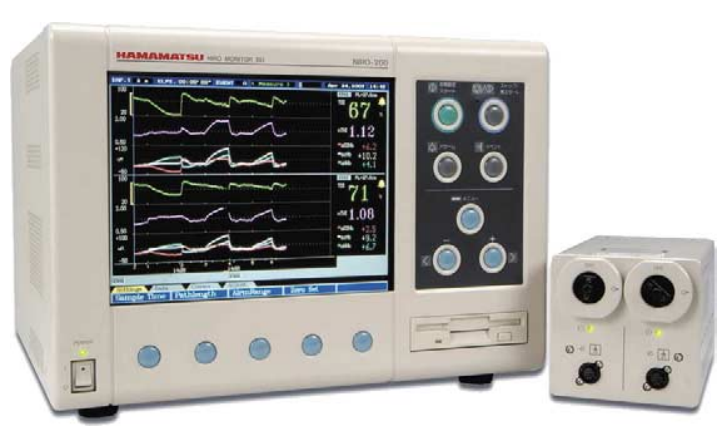

(A)

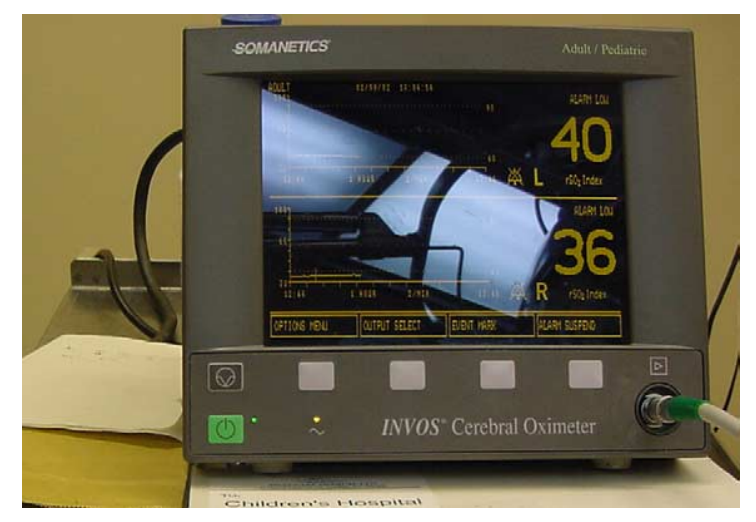

(B)

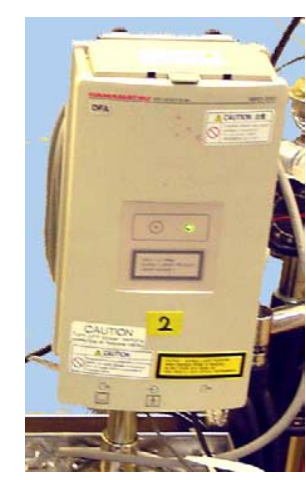

(C)

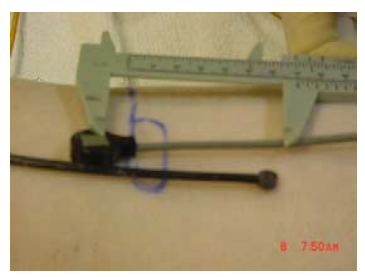

(D)

Fig. 1. Image A: Hamamatsu Photonics KK (Hamamtsu City, Japan) NIRO-200, two channel, three wavelength, $6 \mathrm{~Hz}$ clinical spectrophotometer. Image B: Somanetics Corp (Troy, MI, USA) INVOS-5100 dual channel, two wavelength, 10 sec interval, clinical spectrophotometer. Image C: Four wavelength laser output module of a $6 \mathrm{~Hz}$ Hamamatsu Photonics NIRO-300 clinical spectrophotometer. Image D: The NIRO-300's triple photodiode detector array (left) and emitter prism (right).

and retrograde perfusion occur during the repair of aortic aneurisms, arch replacement, thoracic aortic compromise, or cyanotic and non-cyanotic defects. NIRS has also been an adjunct monitor during vascular grafting, carotid endarterectomy, liver transplantation, resection of anaplastic astrocytomas, resection of glioblastomas, intra-operative blood transfusion, abdominal aortic aneurysmectomy, temporal lobectomy, tracheal extubation, laparoscopic cholecystectomy, fetal monitoring during labor, and urinary tract surgery.

In the diagnostic setting, NIRS has been an adjunct to the evaluation of hydrocephalus, deep vein thrombosis, compartment syndrome, diffuse axonal injury, neuronal signaling in the motor cortex, frontal lobe function in schizophrenia, acute cerebral infarction, psycho physiological assessment of post traumatic stress disorder, venous-arteriolar reflex in gastrocnemius, cognitive function, location of epileptogenic focus, tumor vascularization, lower-back muscle fatigue, G-force induced loss of consciousness, necrotizing fasciitis, Alzheimer's disease, and erectile dysfunction, as well as distinguishing between complex partial seizures and rapid secondarily generalized seizures.

Among treatment regimes, NIRS has monitored haemodialysis, transfusion therapy, endotracheal suctioning, and acupuncture. It has also been used to monitor drug interventions including: aminophylline, 


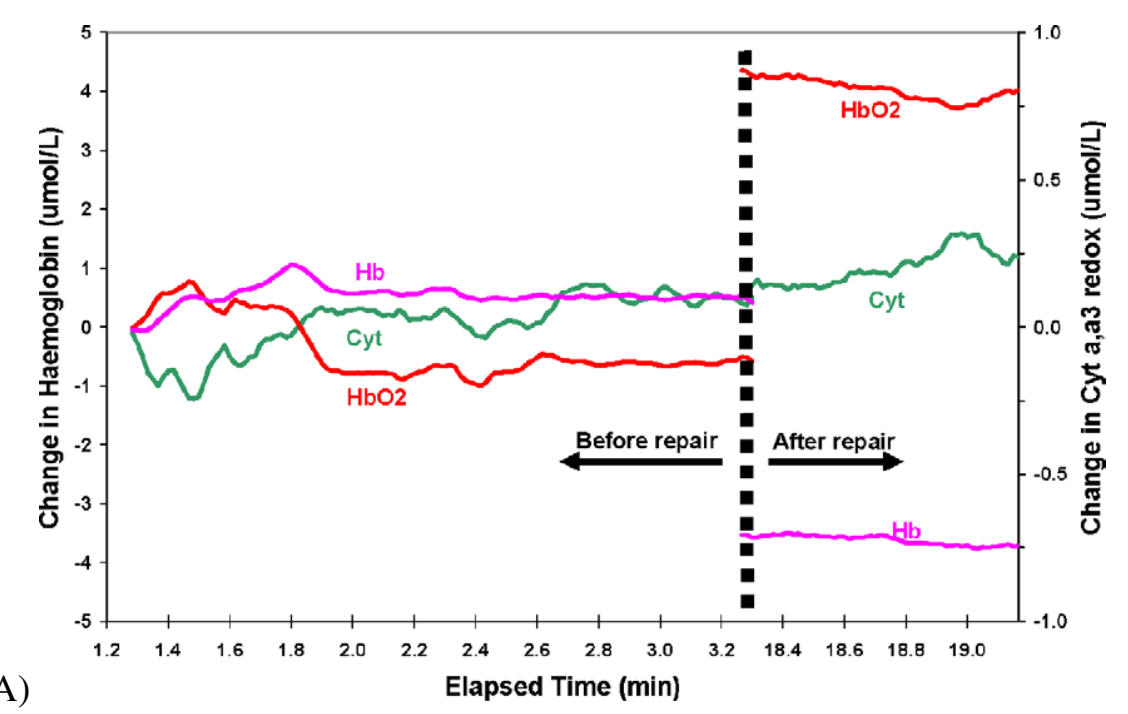

(A)

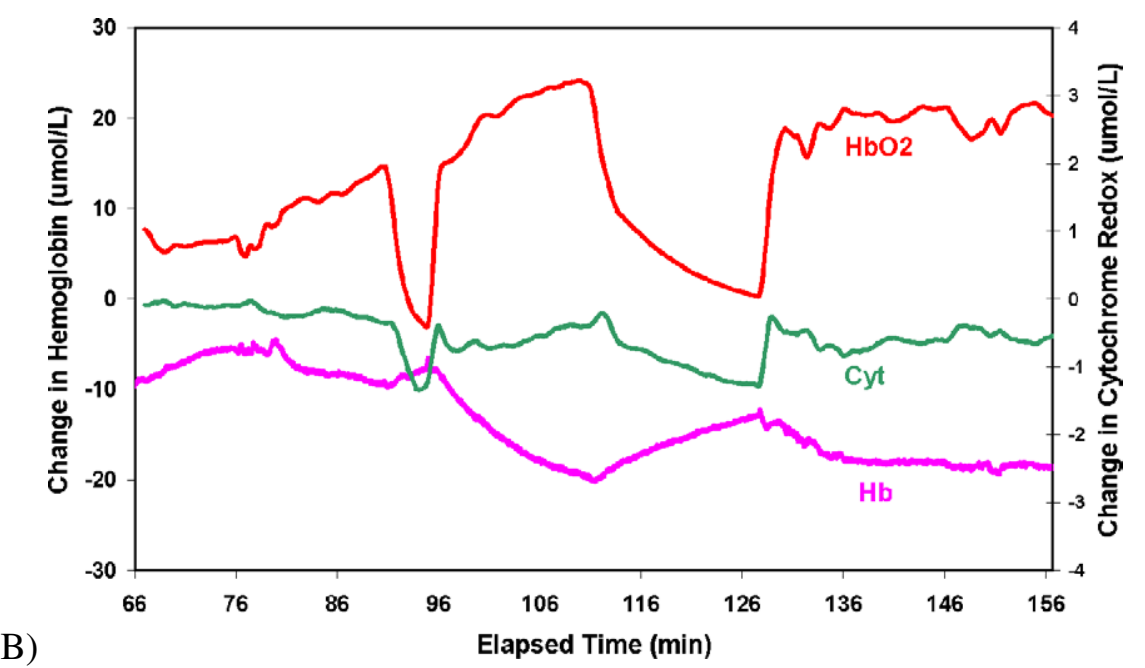

Fig. 2. Graph A: Spinal NIRS during surgical release of the tethered cord in a 6 month old infant. Graph B: Cerebral NIRS in a 15 year old during antegrade perfusion while undergoing heart valve replacement surgery. Graph C: Cerebral NIRS during anesthetized cardiac decremental pacing and radio frequency nerve ablation in a 54 year old. Graph D: Bladder NIRS during non-surgical urinary voiding by an 82 year old with a history of angina and type II diabetes.

surfactant, propofol, ketamine, isoflurane, sevoflurane, vinpocetine, and allopurinol. NIRS has played a vital role in the assessment of recovery from surgical and other treatments including: programmed exercise, abdominal surgery, motor re-organization following stroke, harvesting of the radial artery, and coronary artery grafting.

There are several individual case reports of the use of NIRS in conjunction with malaria, glioma resection, burn wounds, exomphalos, syncope, cerebral vasospasm, retrograde perfusion, intra-arterial thrombolysis, and mitochondrial myopathy.

Although not a clinical application, the same devices and methods have been used in sports science for the study of skiing, speed skating, cycling, weight lifting, rowing, running, walking, endurance exercise, exercise training, and resistance training. 
(C)
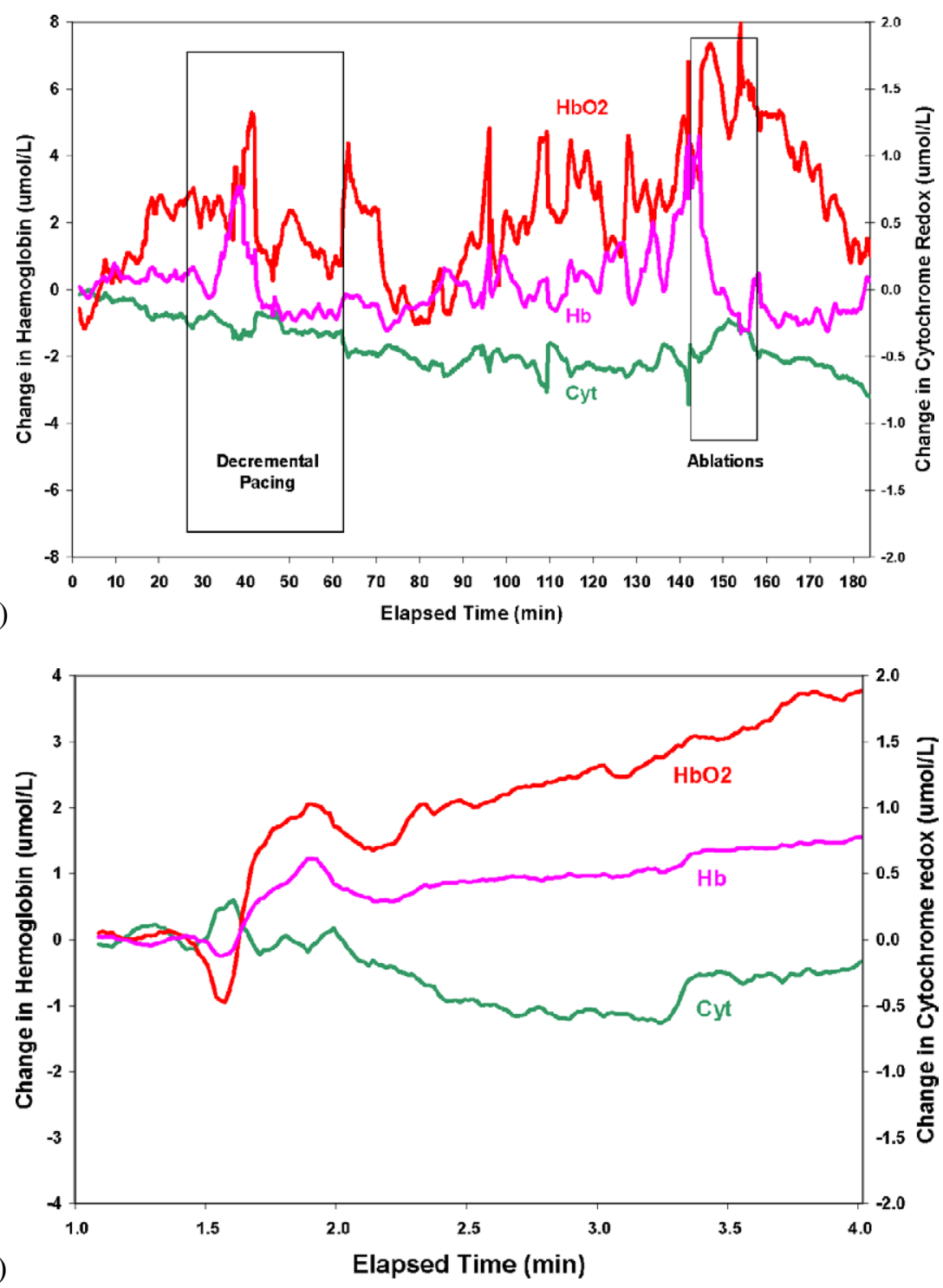

Fig. 2. (Continued).

\section{Algorithms \& assumptions}

The basic underlying formula of many clinical NIRS systems includes the same equation for constant relative rate of growth/decay as used by many other sciences such as bacteria population estimation, Newton's law of cooling, carbon-14 dating, and financial applications, which in the case of NIRS is written as: $I=I_{o} e^{ \pm r t}$, where $I$ is the decreased intensity of the emitted light, $I_{o}$ is the original intensity of the emitted light, $e$ is "Euler's $e$ " (i.e. 2.718282...), $r$ is a constant known as the absorption coefficient specific to the wavelength of light and chromophore being sampled, and $t$ is the linear thickness of the sampling medium. 
The foregoing relates to Lambert's findings of 1760 , but a century later Beer determined that the same formula can be applied to chromophore concentration rather than thickness. Clinical NIRS measurements of light transport depend upon combined thickness and concentration which can be resolved mathematically as $I=I_{o} e^{ \pm r c t}$ and alternatively $I / I_{o}=10^{-\beta c t}$ or $\log \left(I_{o} / I\right)=\beta c t$ where $c$ is the chromophore concentration, and $\beta$ is the reciprocal value of the thickness in centimeters of a one molar solution when the light emerging from the solution is one tenth of the original intensity. These formulae assume that there is only one chromophore and that it is uniformly distributed throughout the sampling medium. However, in clinical applications there are multiple chromophores residing in chaotic fashion within a medium of undetermined thickness. This is resolved using multiple wavelengths of emitted light, at least one per chromophore of interest, and modifying the Beer/Lambert formula given above to replace linear thickness with a correction factor for the separation between emitter and detector in conjunction with a scattering coefficient to account for non-absorbing undetected photons. Thus a matrix solution method is required of the form: $\log \left(I_{o} / I\right)_{\alpha}=\left[\beta_{1} c_{1}+\beta_{2} c_{2}+\beta_{3} c_{3}+\cdots+\beta_{n} c_{n}\right] d P+S$ where $\alpha$ identifies the equation within the matrix consisting of one equation for each wavelength, $d$ is the linear distance between the light emitter and detector, $P$ is a path length correction factor to account for non-linearity, and $S$ is the scattering coefficient [6,7].

Living tissues, however, are not entirely obliging of such formulae. In the case of NIRS it must be assumed that each of chromophores present in the tissue but not accounted for in the matrix solutions are either too weakly absorbing or do not change in concentration. It must also be assumed that the number of scattered photons remains constant, the wavelength dependent path length factor is valid for the given tissue in the given subject, and that the separation between emitter and detector is also constant. A primary assumption is that Euler's " $e$ " realistically mimics the continuous compounding of the loss of photons as biological tissue thickness increases. While the use of " $e$ " is a logical assumption to gain improved accuracy, there is no experimental validation for its use in clinical NIRS. " $e$ " is a standard component of bench-top analytical spectroscopy, but in that setting the chromophore is sampled at a standardized concentration in a standardized volume and is homogenously distributed; therefore, " $e$ " can then be validated to represent the missing photons that cannot be accounted for as being either absorbed by the chromophore or collected by detectors surrounding the sample chamber [1]. In benchtop spectroscopy, a somewhat imperfect " $e$ " can be used as a substitute for the true lost photon count because it is used identically for both the known purified-sample and the comparison unknown-sample. However, the same is not true in clinical NIRS where the known purified-sample data is derived from bench-top non-reactive purified chromophores in a standardized volume, temperature, concentration, and distribution while the comparison unknown-sample data is collected from an unknown volume with heterogeneous distribution and uneven temperatures in a biologically reactive environment that cannot be entirely surrounded by photon detectors.

The inherent assumptions of clinical NIRS using the Beer/Lambert methodology dictate that it can only be used to determine absolute changes in concentration relative to an initial unknown concentration at the start of the data collection. Consequently, it can only be used for intra-subject trials where the data collection is not interrupted and cannot be used for inter-subject trials or interrupted data collections except by comparison of the patterns of change and magnitudes and rates of change [8]. Furthermore, such data collections must have changes resulting from clinical intervention or pathology that are significantly different from those arising from the ongoing uncontrolled variability inherent in the NIRS methodology. For example, when NIRS optodes are placed on the soft tissue of the intact chest an undulating pattern of apparent change in hemoglobin concentration is obtained that corresponds with the cycle of inhalation and expiration. However, such a pattern of change cannot be entirely attributed to 
actual change in concentration because the linear separation and path length between optodes changes with expansion and contraction of the chest cavity during the respiration cycle. In this example, NIRS may be unsuitable for estimation of changes in hemoglobin during normal breathing but could be useful to determine rate of respiration, and could indicate significant changes during breath holding and hypoxic/ischaemic episodes.

\section{Instrumentation \& data collection}

The limitations of conventional NIRS Beer/Lambert methodology have led developers to derive new technologies aimed toward absolute quantification of the chromophore concentrations.

Clinical NIRS instrument designs can be classified by electro-mechanical components, sampling technique, the number of wavelengths, the number of data channels, and the data collection interval(s) (Fig. 1). The electro-mechanical components handle four separate aspects of light: emission, detection, transmission and occlusion. Emitters can be comprised of filtered white light, direct-gap gallium aluminum arsenide semiconductor lasers, or light emitting diodes. Detectors can be incorporated as photon multiplier tubes, micro-channel plate photon multiplier tubes, photo diodes, avalanche photo diodes, charge coupled detector arrays, or synchro-scan streak cameras. Light transmission can be via fibre optic cables, liquid light guides, direct surface contact, or surface stand-off piping. Unwanted radiation can be occluded by optical filters, shielding masks, or chamber darkness. Besides the choice of components in a spectrometer, there are five photonics instrumentation techniques used for acquiring data: continuous wave, spatially resolved, time resolved, intensity modulated, and frequency domain. Common NIRS devices have been designed that utilize one to five or more wavelengths, depending on the intended clinical application and data interpretation capabilities. During operational use, instruments can have either one channel of data collection at a single site, multi-channel collection at diverse sites, or have optical multiplexing for multi-channel collection at a single site. Devices that primarily only measure local hemoglobin reactions at a single site usually have data collection rates slower than $1 \mathrm{~Hz}$, while those including cytochrome redox status or multi-channel capability use $1 \mathrm{~Hz}$ rates or faster.

\section{Chromophores \& controversy}

Clinical NIRS seeks to have significant tissue depth penetration. Consequently, it must operate in the $750 \mathrm{~nm}$ to $950 \mathrm{~nm}$ wavelength range where water has only slight absorbtivity. Unfortunately, this constrains NIRS to use the weakest portions of the hemoglobin and cytochrome spectra rather then those portions with peak absorptivity. In this range, NIRS cannot distinguish circulatory hemoglobin from its intra-cellular counterpart, myoglobin. This has not been of overriding consequence because NIRS is primarily intended for brain monitoring where myoglobin is not present and myoglobin contributes less than $10 \%$ of the signal in muscle studies where it is present. However, this same constraint has led to speculation of whether NIRS can accurately distinguish between hemoglobin and cytochrome.

Each cell in the human body contains between a hundred and a thousand other smaller cells known as mitochondrion. Each host cell and each mitochondrion have their own separate and different DNA. This is an evolutionary symbiosis that seems to have arisen to compensate the natural toxicity of oxygen. While mitochondria use oxygen in the catabolic process of generating life supporting energy from sugars for the host cell, oxygen is also poisonous to the tissues in that it can destroy other life supporting molecules. The mitochondria safely transfer electrons to oxygen thereby stabilizing it in the formation of 


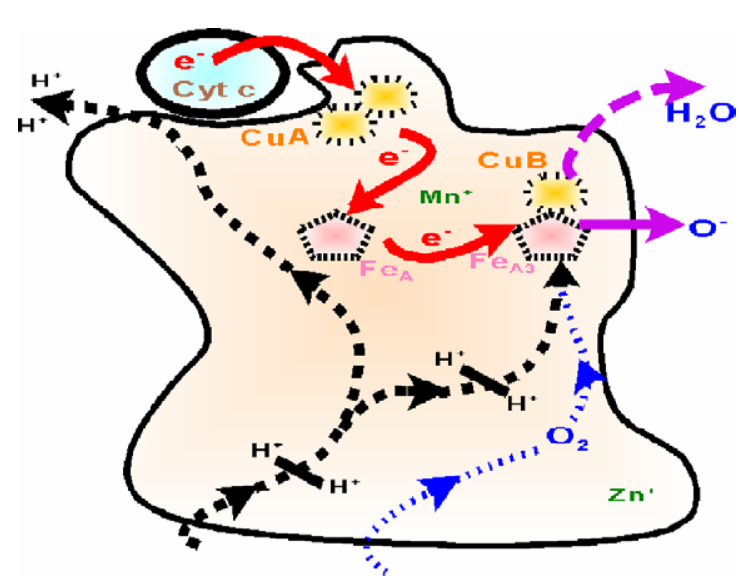

(A)

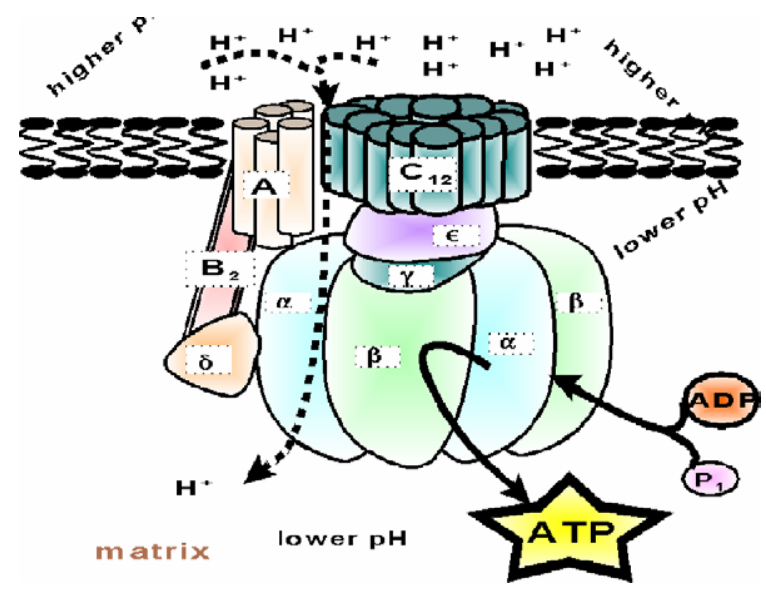

(B)

Fig. 3. Sketch A: Schematic of the electron and proton pathways through the cytochrome a, $\mathrm{a}_{3}$ molecule measured by NIRS. Sketch B: Schematic of the proton pathway through the adenosine triphosphate synthase molecule, which, while rotating brings adenosine diphosphate and phosphate into proximity to yield ATP.

water $\left(\mathrm{H}_{2} \mathrm{O}\right)$ for eventual re-use or removal from the body. Without oxygen the mitochondria would still function to provide energy to the host cell but at a greatly reduced efficiency. Within each mitochondrion, cytochrome $\mathrm{a}, \mathrm{a}_{3}(\mathrm{Cyt})$, measured by NIRS, is the molecular complex that transfers electrons in step wise fashion to create the molecules of $\mathrm{H}_{2} \mathrm{O}$.

During the electron transfer process, protons $\left(\mathrm{H}^{+}\right)$pass through the Cyt complex whenever oxygen enters the Cyt complex. Some protons depart the Cyt complex with oxygen as $\mathrm{H}_{2} \mathrm{O}$, while simultaneously other pairs of $\mathrm{H}^{+}$depart unattached (Fig. 3). The departing $\mathrm{H}^{+}$pairs create an overabundance of $\mathrm{H}^{+}$ near to the adenosine triphosphate synthase molecule (ATPase) neighbouring the Cyt complex. As the surplus $\mathrm{H}^{+}$diffuses through the ATPase complex it causes that molecule to rotate thereby bringing phosphate ions into contact with adenosine di-phosphate (ADP) to form adenosine tri-phosphate (ATP) (Fig. 3). ATP is then free to depart the mitochondrion and distribute within the host cell where it serves to provide energy by releasing the phosphate ion to once again become ADP. The energy provided by ATP is used for maintaining the host's activities and for repairing the host cell's functional components. Thus, the redox status of mitochondrion Cyt measured by NIRS gives insight into overall cell health or compromise.

A mitochondrion is $1 / 14,000$ the size of a typical human cell, and Cyt is $1 / 304,000,000,000$ the size of a typical human cell. Each cell has between 100 and 1600 mitochondria and each mitochondrion has 50,000 Cyt molecules. Cyt transfers between 350 and 750 electrons per second and produces 90 to 190 molecules of $\mathrm{H}_{2} \mathrm{O}$ per second, which consequently leads to the generation of 100 to 300 ATP molecules per second. A typical NIRS $130 \mathrm{ml}$ cerebral sample volume will encompass 300 billion cells, 300,000 billion mitochondria, 15 million-billion cytochrome a, $\mathrm{a}_{3}$ molecules. NIRS will emit one photon for every 9000 Cyt molecules (1700 billion photons emitted per second) and detect 130,000 unabsorbed photons per second.

Concern over the cytochrome signal first arose in 1990 when a collection showing an increase in the oxidized form of cytochrome a, $\mathrm{a}_{3}$ during increased oxygen delivery, a plausible consequence, was instead attributed to "cross-talk" with the hemoglobin signal $[9,10]$. Later, a decrease in the oxidized form of cytochrome a, $\mathrm{a}_{3}$ during decreased blood volume, also a plausible consequence, was instead 
believed to need further validation; and, insignificant or inconsistent cytochrome results during cerebral desaturation raised further scepticism [11,12]. In response, investigators tested the reported cytochrome $\mathrm{a}, \mathrm{a}_{3}$ spectra and calculation algorithms, as well as the likelihood of cross-talk $[6,7,13,14]$. The 1996 Royal Society (London) public symposium on NIRS demonstrated that cytochrome $\mathrm{a}, \mathrm{a}_{3}$ data collections can be free of cross-talk [15]. Ensuing studies demonstrated that some cross-talk might exist in a swine model, was very minimal in a swine model, can be enhanced or diminished in Monte Carlo simulations, and did not exist in 50\% of human trials intended to confirm the Monte Carlo simulations [16-19]. In the laboratory setting using NIRS to detect nano-molar concentrations of Cy 5.5 and Cy 7 fluorochromes, cross-talk between the fluorochromes was assessed, modeled, and then compensated for [20]. The same proficiency has not yet been developed for clinical NIRS; although, it remains inconclusive whether such a utility is needed.

\section{Indices \& ratios}

When clinical NIRS is used to solve only for changes in oxy or de-oxy hemoglobin concentration it is very much akin to the more common-place pulse oximetry. Whereas pulse oximetry provides peripheral capillary oxygenation data and can use the same wavelengths as clinical NIRS, NIRS interrogates greater tissue depths and may either include or exclude pulsatility. Pulse oximetry has gained extensive use in part because its output is a simple display of an index percentage representing arterial hemoglobin oxygen saturation (i.e. $\mathrm{S}_{\mathrm{p}} \mathrm{O}_{2}=$ "peripheral oxygen saturation" or $\mathrm{S}_{\mathrm{a}} \mathrm{O}_{2}=$ "arterial oxygen saturation") with readily recognized ranges for normal and pathologic conditions [21]. Clinical NIRS has sought this same ease-of-use for mixed arterial/venous deep tissue oxygenation status by using spatially resolved spectrometers to express the absolute ratio of oxygenated hemoglobin to total hemoglobin concentration (i.e. $\mathrm{TOI}=$ "tissue oxygenation index", $\mathrm{rSO}_{2}=$ "regional oxygen saturation", $\mathrm{SCO}_{2}=$ "cerebral oxygen saturation") [22].

The NIRS indices have been compared between devices, and found to have different response ranges. Validation trials in comparison with jugular bulb methods have both confirmed and rejected the NIRS indices. Sources of error have been investigated and there have been supporting trials with good correlation to oxygen partial pressure, intracranial pressure, and carbon dioxide reactivity.

The NIRS indices - TOI, and $\mathrm{rSO}_{2}$, are derived from the principles of photon diffusion, time of flight, and spatial resolution rather than those of historical bench top spectroscopy outlined above [22]. The algorithm relies upon replacing relative absorption coefficients used to derive $\mathrm{HbO}_{2}$ and $\mathrm{Hb}$ relative concentrations, with the normalized rate of change in scattering versus wavelength as a constant moderating the differential ratio of attenuation versus emitter-to-detector separation distance, in order to derive absolute $\mathrm{HbO}_{2}$ and $\mathrm{Hb}$ concentrations. Since this algorithm derives absolute $\mathrm{HBO}_{2}$ and $\mathrm{Hb}$, both values can be summated to estimate absolute "total hemoglobin".

TOI is based upon a $1.0 \mathrm{~mm}$ spatial difference between each of three photodiode sensors, while $\mathrm{rSO}_{2}$ is based upon a $10.0 \mathrm{~mm}$ spatial difference between two photodiode sensors. Correlation studies have shown that $\mathrm{TOI}$ and $\mathrm{rSO}_{2}$ are in close agreement during major physiological changes, but less so during minor interventions [23]. The clinical utility of these indices is controversial with similar studies refuting $[24,25]$ and validating $[26,27]$ them. For example, it was found that transcutaneous TOI did not correlate with liver hepatic venous oxygenation in children but did correlate with liver mixed venous oxygenation in piglets. Other investigators have found that, while liver TOI correlates well with central venous oxygen saturation in critically ill children, there are large inter-subject differences. 
The intent of having the NIRS indices is to enable absolute measures thereby permitting inter- and intra-subject comparisons over separate data collection occasions. However, such comparisons are also possible with the Beer-Lambert derived $\mathrm{HbO}_{2}, \mathrm{Hb}$, and Cyt data if both subjects are connected simultaneously to the same device, or if multiple organ data sets are collected simultaneously in the same subject, or if the NIRS device is not re-initialized between data collections in the same subject. Clinically, the indices display only one key parameter to the care providers so that there can be quick determination of the patient's well being. This is necessary because nurses and doctors now have an overwhelming array of complex measurements arising from many other technologies. However, at this time there are no benchmark studies establishing the pathologic limits for the NIRS indices. For example, practice guidelines such as "a TOI/rSO $\mathrm{rO}_{2}$ of (e.g.) percent, lasting (e.g.) minutes, leads to irreversible impairment" have not yet been established. In its current form, one team of investigators has recently found that TOI does not provide additional practical information for clinical management [28].

\section{Blood flow \& blood volume}

Blood flow rate is one of the most important vital signs, but it cannot readily be measured noninvasively. An existing technology, transcutaneous Doppler ultrasound, is able to non-invasively estimate the surface speed of blood streams, but cannot associate the measurement to the transit volume. Instead, NIRS algorithms have been developed to estimate proportional blood flow rate and transit volume based on the Fick principle of differences in tracer concentrations entering and exiting an organ [29]. NIRS blood flow units are expressed as ml blood $\times 100 \mathrm{~g}_{\text {tissue }}^{-1} \times$ minute $^{-1}$ and NIRS blood volume units are expressed as $\mathrm{ml}_{\text {blood }} \times 100 \mathrm{~g}_{\text {tissue }}^{-1}$. Early experiments sought to use a sudden change in arterial oxygen saturation as the tracer bolus. However, the technique required subjective identification of the point in time when the arterial bolus began to transit into the venous system and this led to irreproducible results and routine exclusion of large numbers of data sets. The technique was revised using indocyanine green as the tracer bolus to establish transit time, and further revised using spatial resolution to create a flow index. Recently, the indocyanine method has been rejected by comparison to Xenon 133 clearance, but accepted in comparison with perfusion weighted MRI [30,31].

Some investigators use the tissue hemoglobin index (THI) to represent blood volume [32]. Since the spatial resolution algorithm (described above), derives absolute $\mathrm{HBO}_{2}$ and $\mathrm{Hb}$, these parameters can be summated to estimate absolute "total hemoglobin", i.e. THI; however, since a single normalized scattering term did not exist for "total hemoglobin" in order to derive THI independently, then THI is designated as a relative index because its discrepancy with the absolute independent measure cannot be determined. THI is seldom reported in the literature; although it has been correlated with SPECT as an indicator of blood volume change [33].

\section{Recent advances}

(At the time of writing this survey includes only the period from December 2004 to September of 2005.)

For those interested in developing their own NIRS devices or software algorithms, a new review of hemoglobin extinction coefficients has been published specifically for those working in the field of tissue spectroscopy [34]. 
There was only one report of using NIRS to study cytochrome c oxidase in humans. It showed that there were no significant changes in redox status during the brief period of ischemia that occurs during surgical cardiac defibrillator replacement [35]. Two swine studies sought to gain insight into the differences in cytochrome redox status results seen between previous investigators. Among these, one was the first report of simultaneously monitoring brain, spine, and limb muscle [36]; while the other explored the influences of combinations of the ranges in temperature, $\mathrm{pH}$, hematocrit, and glucose [37]. Two studies of rat liver surgical techniques used NIRS cytochrome $\mathrm{c}$ oxidase redox status to indicate beneficial tissue well being [38,39]. One swine study was the first report to base its interpretation on TOI, and THI in combination with cytochrome c oxidase without including changes in $\mathrm{HbO}_{2}$ and/or $\mathrm{Hb}$ [40]. An in vitro study used the redox aspects of cytochrome $\mathrm{c}$ oxidase to better understand how near infrared therapy promotes wound healing [41].

This year saw the first report of NIRS in conjunction with modulation based direct current magnetoencephalography (DC-MEG) to monitor low-amplitude magnetic fields arising not only from injured tissue, but also generated by functional cortical activation [42].

The ongoing use of NIRS to study muscle tissue has now been enhanced by modeling studies that indicate subject-to-subject variation in overlying fat thickness can be ignored if the fat thickness is less than $5 \mathrm{~mm}$ [43]. Similarly, those involved in cerebral oxygenation monitoring during surgery now have benefit of a NIRS interpretation matrix to guide brief interventions to optimize protection strategies [44].

All NIRS investigators will be interested in a new approach that uses Wiener filtering to suppress movement artefact in NIRS cerebral studies [45]. The current report demonstrates a useful post collection analysis technique and proposes Kalman filtering as a means for real time suppression. A similarly relevant study has validated a new algorithm that removes the influence of skin pigmentation (melanin) from NIRS collections [46].

In the hardware realm, a new system has been validated that utilizes 4 emitters and 32 detectors on each breast for simultaneous dual breast NIRS tomography to distinguish normal and cancerous tissues [47].

\section{Summary}

More than a dozen commercial clinical NIRS devices are available using the $750 \mathrm{~nm}$ to $950 \mathrm{~nm}$ wavelength electromagnetic range to non-invasively measure either oxygenated hemoglobin, de-oxygenated hemoglobin, oxidized minus reduced cytochrome $\mathrm{a}_{3} \mathrm{a}_{3}$, and blood flow or blood volume. It has been used to interrogate more than a dozen different organs/tissue types in more than 90 clinical conditions.

Research continues towards evolving NIRS systems capable of absolute inter-subject comparisons; and, there is now broader acceptance of NIRS' capability for measuring the patterns of change in cytochrome $\mathrm{a}, \mathrm{a}_{3}$ redox status. The spatially resolved indices (TOI, $\mathrm{rSO}_{2}$, etc.) used by some NIRS devices have as many rejected validation trials as accepted ones. The NIRS relative hemoglobin measurements are widely accepted.

Near infrared spectroscopy has proven itself to be a useful monitoring tool in the clinical setting, and has great potential for further uses in diverse diagnostic and treatment applications.

\section{References}

[1] A.I. Vogel, A Text-Book of Quantitative Inorganic Analysis Including Elementary Instrumental Analysis, 3rd edn, Longmans Green \& Co., London, 1961, pp. 740-742. 
[2] R.N. Jones, Analytical applications of vibrational spectroscopy, a historical review, in: Chemical, Biological, and Industrial Applications of Infrared Spectroscopy, J.R. During, ed., John Wiley and Sons, New York, 1985, pp. 1-43.

[3] B. Chance, C.E. Cooper, D.T. Delpy and E.O.R. Reynolds, Near-infrared spectroscopy and imaging of living systems, Philos. Trans. R. Soc. Lond. B Biol. Sci. 352(1354) (1997), 643-761.

[4] T. Tsukihara, H. Aoyama, E. Yamashita, T. Tomizaki, H. Yamaguchi, K. Shinzawa-itch, R. Nakashima, R. Yaono and S. Yoshikawa, Structures of metal sites of oxidized bovine heart cytochrome c oxidase at $2.8 \AA$, Science 269 (1995), 1069-1074.

[5] A.D. Edwards, J.S. Wyatt, C. Richardson, D.T. Delpy, M. Cope and E.O. Reynolds, Cotside measurement of cerebral blood flow in ill newborn infants by near infrared spectroscopy, Lancet 2(8614) (1988), 770-771.

[6] R.E. Gagnon, F.A. Gagnon and A.J. Macnab, Comparison of 13 published cytochrome c oxidase near-infrared spectroscopy algorithms, Eur. J. Appl. Physiol. 74(6) (1996), 487-495.

[7] R.E. Gagnon and A.J. Macnab, C/C++ Coding for matrix pseudo inverses in clinical near infrared spectroscopy, Comput. Methods Biomech. Biomed. Engin. 1(2) (1998), 69-86.

[8] R.E. Gagnon, A.J. Macnab, F.A. Gagnon, D. Blackstock and J.G. LeBlanc, Comparison of two spatially resolved NIRS oxygenation indices, J. Clin. Monit. Comput. 17(7-8) (2002), 385-391.

[9] O. Pryds, G. Greisen, L.L. Skov and B. Friis-Hansen, Carbon dioxide-related changes in cerebral blood volume and cerebral blood flow in mechanically ventilated preterm neonates: comparison of near infrared spectrophotometry and 133xenon clearance, Pediatr. Res. 27(5) (1990), 445-449.

[10] L. Skov, O. Pryds and B. Friis-Hansen, Near infrared spectrophotometry - a non-invasive, continuous method for monitoring of cerebral status in newborn infants [Article in Danish], Ugeskr. Laeger 152(23) (1990), 1646-1650.

[11] L. Skov and G. Greisen, Apparent cerebral cytochrome aa3 reduction during cardiopulmonary bypass in hypoxaemic children with congenital heart disease. A critical analysis of in vivo near-infrared spectrophotometric data, Physiol. Meas. 15(4) (1994), 447-457.

[12] Y.A. Wickramasinghe, P. Rolfe, K. Palmer and S.A. Spencer, Investigation of neonatal brain cytochrome redox by NIRS, Brain Res. Dev. Brain Res. 89(2) (1995), 307-308.

[13] A.J. Macnab and R.E. Gagnon, Potential sources of discrepancies between living tissue near infrared spectroscopy algorithms, Anal. Biochem. 236 (1996), 375-377.

[14] S.J. Matcher, C.E. Elwell, C.E. Cooper, M. Cope and D.T. Delpy, Performance comparison of several published tissue near-infrared spectroscopy algorithms, Anal. Biochem. 227 (1995), 54-68.

[15] C.E. Cooper and R. Springett, Measurement of cytochrome oxidase and mitochondrial energetics by near-infrared spectroscopy, Philos. Trans. R. Soc. Lond. B Biol. Sci. 352(1354) (1997), 669-676.

[16] T. Sakamoto, R.A. Jonas, U.A. Stock, S. Hatsuoka, M. Cope, R.J. Springett and G. Nollert, Utility and limitations of near-infrared spectroscopy during cardiopulmonary bypass in a piglet model, Pediatr. Res. 49(6) (2001), 770-776.

[17] R. Springett, J. Newman, M. Cope and D.T. Delpy, Oxygen dependency and precision of cytochrome oxidase signal from full spectral NIRS of the piglet brain, Am. J. Physiol. Heart Circ. Physiol. 279(5) (2000), H2202-H2209.

[18] K. Uludag, M. Kohl, J. Steinbrink, H. Obrig and A. Villringer, Cross talk in the Lambert-Beer calculation for near-infrared wavelengths estimated by Monte Carlo simulations, J. Biomed. Opt. 7(1) (2002), 51-59.

[19] K. Uludag, J. Steinbrink, M. Kohl-Bareis, R. Wenzel, A. Villringer and H. Obrig, Cytochrome-c-oxidase redox changes during visual stimulation measured by near-infrared spectroscopy cannot be explained by a mere cross talk artefact, Neuroimage 22(1) (2004), 109-119.

[20] M.A. Funovics, H. Alencar, H.S. Su, K. Khazaie, R. Weissleder and U. Mahmood, Miniaturized multichannel near infrared endoscope for mouse imaging, Mol. Imaging 2(4) (2003), 350-357.

[21] T. Aoyagi, Pulse oximetry: its invention, theory, and future, J. Anesth. 17(4) (2003), 259-266.

[22] S. Suzuki, S. Takasaki, T. Ozaki and Y. Kobayashi, A tissue oxygenation monitor using NIR spatially resolved spectroscopy, Proc. SPIE 3597 (1999), 582-592.

[23] R.E. Gagnon, A.J. Macnab, F.A. Gagnon, D. Blackstock and J.G. LeBlanc, Comparison of two spatially resolved NIRS oxygenation indices, J. Clin. Monit. Comput. 17(7-8) (2002), 385-391.

[24] N. Nagdyman, T. Fleck, S. Schubert, P. Ewert, B. Peters, P.E. Lange and H. Abdul-Khaliq, Comparison between cerebral tissue oxygenation index measured by near-infrared spectroscopy and venous jugular bulb saturation in children, Intensive Care Med. 31(6) (2005), 846-850.

[25] K. Yoshitani, M. Kawaguchi, M. Iwata, N. Sasaoka, S. Inoue, N. Kurumatani and H. Furuya, Comparison of changes in jugular venous bulb oxygen saturation and cerebral oxygen saturation during variations of haemoglobin concentration under propofol and sevoflurane anaesthesia, Br. J. Anaesth. 94(3) (2005), 341-346.

[26] M.B. Kim, D.S. Ward, C.R. Cartwright, J. Kolano, S. Chlebowski and L.C. Henson, Estimation of jugular venous O2 saturation from cerebral oximetry or arterial $\mathrm{O}_{2}$ saturation during isocapnic hypoxia, J. Clin. Monit. Comput. 16(3) (2000), 191-199. 
[27] N. Shimizu, F. Gilder, B. Bissonnette, J. Coles, D. Bohn and K. Miyasaka, Brain tissue oxygenation index measured by near infrared spatially resolved spectroscopy agreed with jugular bulb oxygen saturation in normal pediatric brain: a pilot study, Childs Nerv. Syst. 21(3) (2005), 181-184.

[28] M. Weiss, G. Schulz, I. Teller, A. Dullenkopf, A. Kolarova, H. Sailer, C.M. Dillier, H.U. Bucher, A.C. Gerber and O. Baenziger, Tissue oxygenation monitoring during major pediatric surgery using transcutaneous liver near infrared spectroscopy, Paediatr. Anaesth. 14(12) (2004), 989-995.

[29] S.S. Kety and C.F. Schmidt, The nitrous oxide method for the quantitative determination of cerebral blood flow in man: theory, procedure, and normal values, J. Clin. Inves. 27 (1948), 476-483.

[30] R.D. Rothoerl, K.M. Schebesch, R. Faltermeier, C. Woertgen and A. Brawanski, Lack of correlation between xenon133 and near infrared spectroscopy/indocyanine green rCBF measurements, Neurol. Res. 25(5) (2003), 528-532.

[31] E. Keller, A. Nadler, H. Alkadhi, S.S. Kollias, Y. Yonekawa and P. Niederer, Noninvasive measurement of regional cerebral blood flow and regional cerebral blood volume by near-infrared spectroscopy and indocyanine green dye dilution, Neuroimage 20(2) (2003), 828-839. (Erratum for equation 5 is given in: Neuroimage 22(3) (2004), 1428.)

[32] B. Bein, E. Cavus, V. Dorges, K.H. Stadlbauer, P.H. Tonner, M. Steinfath and J. Scholz, Arginine vasopressin reduces cerebral oxygenation and cerebral blood volume during intact circulation in swine - a near infrared spectroscopy study, Eur. J. Anaesthesiol. 22(1) (2005), 62-66.

[33] K. Yamamoto, T. Komiyama, T. Miyata, T. Kitagawa, T. Momose, H. Shigematsu and H. Nagawa, Contralateral stenosis as a risk factor for carotid endarterectomy measured by near infrared spectroscopy, Int. Angiol. 23(4) (2004), 388-393.

[34] J.G. Kim, M. Xia and H. Liu, Extinction coefficients of hemoglobin for near-infrared spectroscopy of tissue, IEEE Eng. Med. Biol. Mag. 24(2) (2005), 118-121.

[35] E. McNeill, R.E. Gagnon, J.E. Potts, J.A. Yeung-Lai-Wah, C.R. Kerr and S. Sanatani, Cerebral oxygenation during defibrillator threshold testing of implantable cardioverter defibrillators, Pacing Clin. Electrophysiol. 28(6) (2005), 528-533.

[36] R.E. Gagnon, A.J. Macnab, F.A. Gagnon and J.G. Leblanc, Brain, spine, and muscle cytochrome Cu-A redox patterns of change during hypothermic circulatory arrest in swine, Comp. Biochem. Physiol. A Mol. Integr. Physiol. 141(3) (2005), 264-270.

[37] R.E. Gagnon, F.A. Gagnon, A.J. Macnab and J.G. LeBlanc, Temperature, hematocrit, pH, and glucose 4-way ANOVA of cytochrome c oxidase redox status during systemic cold circulatory arrest in swine, Metab. Brain. Dis. 20(2) (2005), $105-113$.

[38] H. Mitsuta, H. Ohdan, K. Mizunuma, T. Itamoto and T. Asahara, Near-infrared spectroscopic analysis of hepatic hemodynamics and oxygen metabolism after extensive hepatectomy, Med. Sci. Monit. 10(10) (2004), BR356-BR361.

[39] R.S. Koti, W. Yang, G. Glantzounis, A. Quaglia, B.R. Davidson and A.M. Seifalian, Effect of ischaemic preconditioning on hepatic oxygenation, microcirculation and function in a rat model of moderate hepatic steatosis, Clin. Sci. (Lond.) 108(1) (2005), 55-63.

[40] B. Bein, E. Cavus, V. Dorges, K.H. Stadlbauer, P.H. Tonner, M. Steinfath and J. Scholz, Arginine vasopressin reduces cerebral oxygenation and cerebral blood volume during intact circulation in swine - a near infrared spectroscopy study, Eur. J. Anaesthesiol. 22(1) (2005), 62-66.

[41] M.T. Wong-Riley, H.L. Liang, J.T. Eells, B. Chance, M.M. Henry, E. Buchmann, M. Kane and H.T. Whelan, Photobiomodulation directly benefits primary neurons functionally inactivated by toxins: role of cytochrome c oxidase, J. Biol. Chem. 280(6) (2005), 4761-4771.

[42] B.M. Mackert, The discovery of slowness - recent progress in DC-MEG research, Neurol. Clin. Neurophysiol. 30 (2004), 41.

[43] Y. Yang, O.O. Soyemi, M.R. Landry and B.R. Soller, Influence of a fat layer on the near infrared spectra of human muscle: quantitative analysis based on two-layered Monte Carlo simulations and phantom experiments, Opt. Express. 13(5) (2005), 1570-1579.

[44] G. Schwarz, G. Litscher, P.A. Delgado and G.E. Klein, An NIRS matrix for detecting and correcting cerebral oxygen desaturation events during surgery and neuroendovascular procedures, Neurol. Res. 27(4) (2005), 423-428.

[45] M. Izzetoglu, A. Devaraj, S. Bunce and B. Onaral, Motion artifact cancellation in NIR spectroscopy using Wiener filtering, IEEE Trans. Biomed. Eng. 52(5) (2005), 934-938.

[46] O.O. Soyemi, M.R. Landry, Y. Yang, P.O. Idwasi and B.R. Soller, Skin color correction for tissue spectroscopy: demonstration of a novel approach with tissue-mimicking phantoms, Appl. Spectrosc. 59(2) (2005), 237-244.

[47] C.H. Schmitz, D.P. Klemer, R. Hardin, M.S. Katz, Y. Pei, H.L. Graber, M.B. Levin, R.D. Levina, N.A. Franco, W.B. Solomon and R.L. Barbour, Design and implementation of dynamic near-infrared optical tomographic imaging instrumentation for simultaneous dual-breast measurements, Appl. Opt. 44(11) (2005), 2140-2153. 


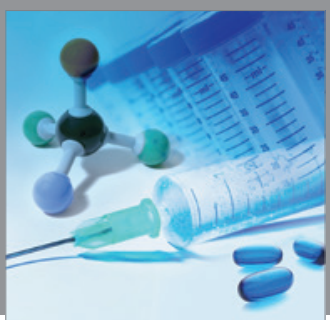

International Journal of

Medicinal Chemistry

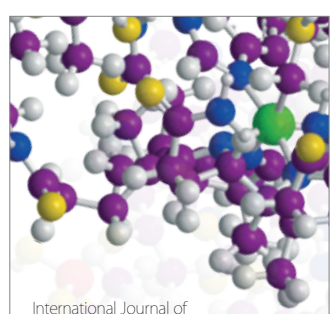

Carbohydrate Chemistry

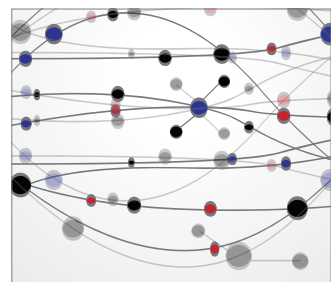

The Scientific World Journal
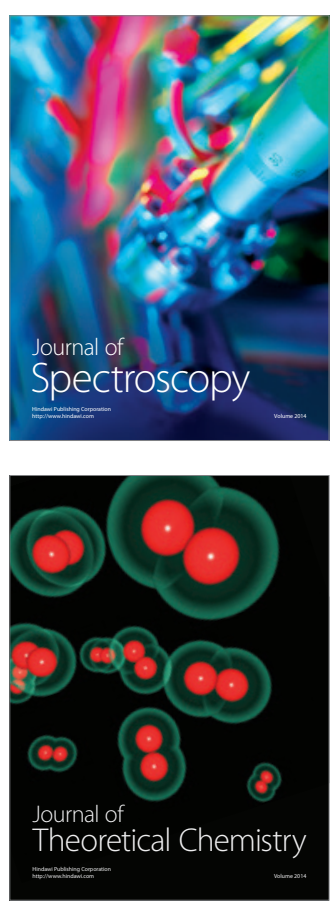
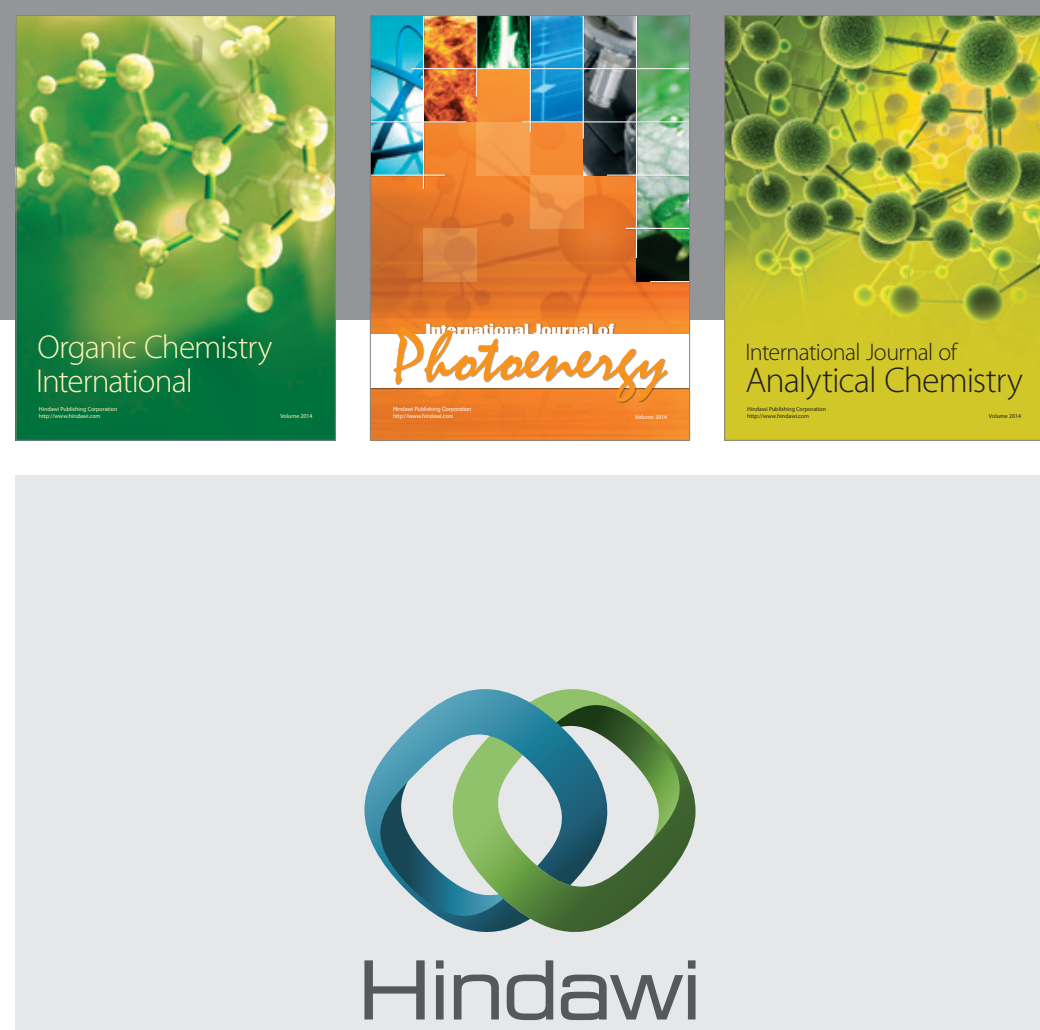

Submit your manuscripts at

http://www.hindawi.com
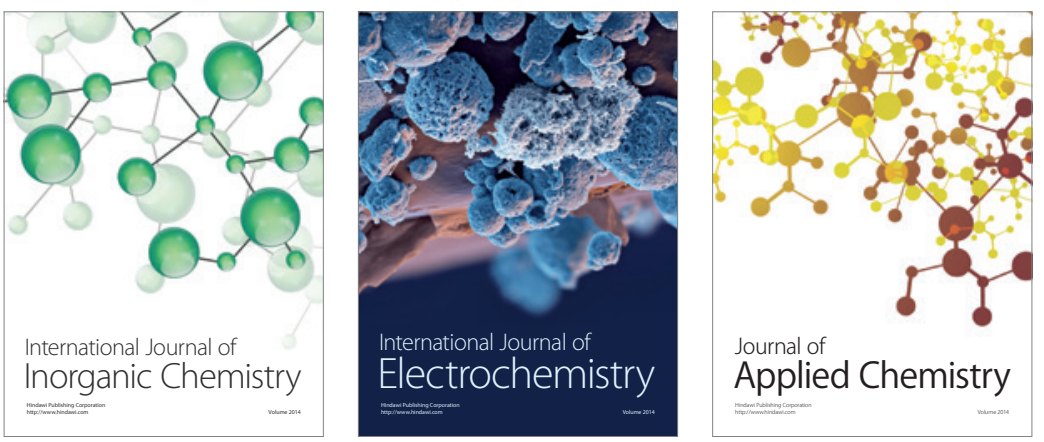

Journal of

Applied Chemistry
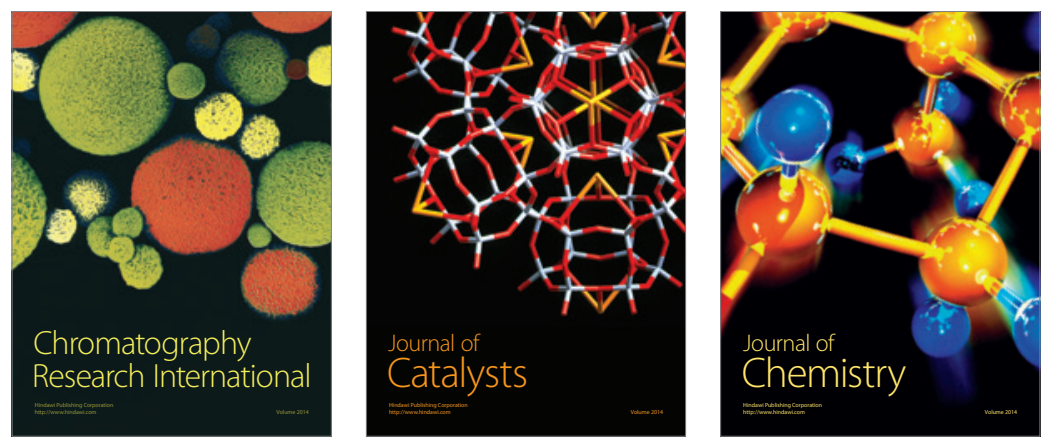
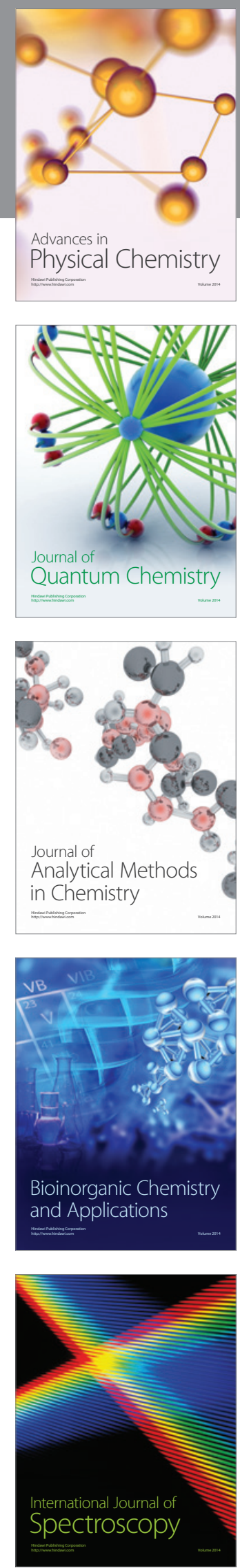